\title{
Preparation and characterization of electrospun alginate nanofibers loaded with ciprofloxacin hydrochloride
}

Agnieszka Kyzio1 $^{1 *}$, Justyna Michna ${ }^{1}$, Ivan Moreno ${ }^{2}$, Enrique Gamez ${ }^{2}$, Silvia Irusta ${ }^{2,3}$

${ }^{1}$ Jagiellonian University, Faculty of Chemistry, Gronostajowa 2, 30-387 Kraków, Poland

${ }^{2}$ Department of Chemical Engineering, Nanoscience Institute of Aragon (INA), University of Zaragoza, 50018 Zaragoza, Spain

${ }^{3}$ Networking Research Center on Bioengineering, Biomaterials and Nanomedicine, CIBERBBN, 50018 Zaragoza, Spain

*Author for correspondence: Agnieszka Kyzioł, Faculty of Chemistry, Jagiellonian University, 30-060 Cracow, Tel. +48-12-6862484, Fax: +48-12-6340515, e-mail: kyziol@chemia.uj.edu.pl

\begin{abstract}
Alginate nanofibers were prepared in the presence of poly(ethylene oxide) (PEO), a surfactant Pluronic F-127, and a model drug (ciprofloxacin hydrochloride, $\mathrm{CpHCl}$ ), all mixed prior to electrospinning. It was demonstrated that addition of a carrier polymer (PEO) and a small amount of surfactant are necessary to obtain uniform alginate fibers with cylindrical shape and regular morphology. Importantly, PEO was completely removed from the resulting nanofibers during crosslinking and stabilization post-treatment. The stable alginate fibers loaded with $\mathrm{CpHCl}$ were examined by scanning electron microscopy and the average diameter of the fibers ranged from $109 \mathrm{~nm}$ (unloaded fibers) to $161 \mathrm{~nm}$ (loaded fibers). The release of a studied antibiotic from the nanofibers, characterized by a final loading efficiency of $51 \%$, was tested in physiological conditions. It was revealed that $c a$. $24 \%$ of $\mathrm{CpHCl}$ is released during first $20 \mathrm{~h}$ with combined transport mechanism, however with the predominant contribution of Fickian diffusion.
\end{abstract}

Keywords: nanofibers; alginate; ciprofloxacin hydrochloride; drug release 


\section{Introduction}

Electrospinning is a simple and versatile method for fabrication of continuous and uniform polymeric nanofibers. Various synthetic polymers (e.g. PCL, PLA, PGLA, etc.) have been successfully electrospun into ultrafine fibers [1-3]. While natural polymers (e.g. chitosan, sodium alginate, hyaluronic acid, etc.) usually need to be electrospun in blend form by mixing them with synthetic polymers such as PEO, PVA or PVP, which improves their electrospinning processability [4-8]. High specific area, tunable pore size, controlled mechanical properties, and their ability to interact with cells in a manner which mimics the natural ECMs mainly cause that fibrous materials are finding an increasing range of applications, including biomedical areas e.g. antibacterial fibers for wound dressings [9], scaffolds for tissue engineering (i.e. skin, bone, cartilage and cardiac tissue) [5, 10, 11], drug delivery systems $[12,13]$, medical implants, protective textiles, filtration systems, etc. [7, 8, 14-17].

Alginate was found to possess many significant and desirable properties as material for wound dressing such as decent water absorptivity, conformability, optimal water vapor transmission rate, and mild antiseptic properties coupled with non-toxicity, nonimmunogenicity, and biocompability $[4,8,18]$. In particular, when alginate is combined with well-known biocides (e.g. antibiotics, metal nanoparticles, etc.) it forms an effective antibacterial wound dressing, that offers many advantages including hemostatic capability, gel-forming ability upon absorption of wound exudates and last but not least well-defined drug delivery system. For instance, it has been suggested that alginate dressings (e.g. Kaltostat $\left.{ }^{\circledR}\right)$ can enhance wound healing by stimulating monocytes to produce elevated levels of cytokines such as interleukin-6 and tumor necrosis factor- $\alpha$. Production of these cytokines at wound sites results in pro-inflammatory factors that are advantageous to wound healing [19].

Electrospinning of alginate is still a challenging task, since sodium alginate is a polyelectrolyte having high conductivity and surface tension. Even though alginate can form solutions with a wide range of viscosity, in this case viscosity is not a limiting factor but the repulsive force among the polyanions are the key factor hindering electrospinning of sodium alginate [20]. These repulsive forces can be reduced by interactions between PEO and sodium 
alginate occurring after blending. Thus, it allows successful electrospinning of sodium alginate/PEO blends [6, 7, 21, 22].

In this paper, we present a simple method of electrospinning of sodium alginate nanofibers loaded with ciprofloxacin hydrochloride, a fluoroquinolone antibiotic that is widely used for wound healing applications. Alginate blended with poly(ethylene oxide) was electrospun into fibers loaded with the above-mentioned antibiotic from an aqueous solution. Thus, the cytotoxicity, usually involved in preparing the electrospun mats by using toxic solvents, was minimized in this method due to application of only biocompatible materials. Effect of an additional polymer, a surfactant, and alginate concentration on formation of fibers was studied in detail. Moreover, kinetics of antibiotic release from well-characterized stable alginate hybrid fibers was studied in a physiological fluid (phosphate-buffered saline, $\mathrm{pH}=7.4)$.

\section{Experimental section}

\subsection{Materials}

Sodium alginate (AL), extracted from seaweed Macrocystis pyrifera, was purchased from Sigma-Aldrich. According to manufacturer, a viscosity of a $1 \% \mathrm{AL}$ solution at $25^{\circ} \mathrm{C}$ is 5.0 - 40.0 cP. Poly(ethylene oxide) (PEO, $\mathrm{M}_{\mathrm{W}}$ 100, 600, 1000 and $2000 \mathrm{kDa}$ ), Triton X-100, Pluronic F-127, ciprofloxacin hydrochloride $(\mathrm{CpHCl})$, ethanol and calcium chloride were also purchased from Sigma-Aldrich. All reagents were used without any further purification.

\subsection{Fabrication of alginate-based electrospun fibers loaded with ciprofloxacin hydrochloride}

Sodium alginate, PEO and ciprofloxacin hydrochloride were dissolved in water at the desired final concentrations in ranges from: 1-5 wt.\%, 1.5-3 wt.\% and 0.02-0.48 wt.\% (0.2$4.8 \mathrm{mg} / \mathrm{ml}$ ), respectively. Then, the surfactants: Triton X-100 or Pluronic F-127 were added at final concentrations of $0.5-1$ wt.\%. The prepared mixtures were stirred overnight at room temperature.

An Yflow 2.2 D500 electrospinner with a coaxial setmp was used to obtain the fibers that were collected on a plate covered with Parchment paper for easy removal of mats. The polymer solution was pumped through a syringe with a 22 gauge needle. The pump was 
working at flow rate of $0.1-1.0 \mathrm{ml} / \mathrm{h}$. The distance between the end of the needle and the collector plate was fixed at $15-20 \mathrm{~cm}$. A voltage was varied from 6 to $10 \mathrm{kV}$ until a stable Taylor cone was achieved. All nanofibers were obtained at room temperature and relative humidity 30-50\%.

2.3. Crosslinking and stabilization of alginate-based electrospun fibers loaded with ciprofloxacin hydrochloride

After electrospinning fibers were mechanically removed from the collector plate and ionically crosslinked. Fibers were soaked in ethanol $(1 \mathrm{~min})$, followed by calcium chloride solution (2 wt.\%) in 1:5 ethanol:water $(10 \mathrm{~min})$. Subsequently, fibers were removed from calcium chloride solution and lyophilized.

\subsection{Characterization of the electrospun fibers}

Fiber mats were characterized by IR spectroscopy (Vertex 70, Bruker) using an ATR Golden Gate accessory. Furthermore, a scanning electron microscopy (SEM, FEI XL30) was applied for fibers characterization. Fiber diameters and standard deviations on 200 fibers per sample were measured using ImageJ program.

\subsection{Ciprofloxacin hydrochloride encapsulation efficiency}

The ciprofloxacin concentration was determined by UV-VIS spectrophotometry at $277 \mathrm{~nm}$ (Lambda 35, Perkin Elmer). A standard calibration curve for ciprofloxacin were prepared. Encapsulation efficiency was calculated by the following equation (1):

$L E \%=\frac{L}{T} \times 100 \%$

where $\mathrm{T}$ and $\mathrm{L}$ are respectively total and loaded amount of ciprofloxacin. Total amount of drug was recognized as an initial concentration of $\mathrm{CpHCl}$ in the synthesis solution. The loaded $\mathrm{CpHCl}$ concentration was calculated according to the calibration curve equation after dissolving the as-spun fiber in water. 
The drug lost during the crosslinking was measured in ethanol and $\mathrm{CaCl}_{2}$ solution used in the process and the final ciprofloxacin load was obtain by difference between the amount remaining in the as-spun fibers according to the calculated LE\% and the loss in the crosslinking solutions.

\subsection{Release of ciprofloxacin hydrochloride}

The release process was carried out in a continuous mode using a Shimadzu LC-10AT VP syringe pump. Crosslinked fibers were put into the syringe and rinsed with phosphatebuffered saline (PBS) at $37^{\circ} \mathrm{C}$. Rinsed solution was collected in $1.5 \mathrm{ml}$ eppendorf vials in different time intervals (1-10min; 20-60min; $1 \mathrm{~h}-6 \mathrm{~h})$. The flow rate was $1 \mathrm{ml} / \mathrm{h}$, time of the whole ciprofloxacin hydrochloride release was $6 \mathrm{~h}$. Collected samples were analyzed by UVVIS spectrophotometry at $277 \mathrm{~nm}$ (Lambda 35, Perkin Elmer). Amount of the released CpHCl was determined from ciprofloxacin calibration curve.

\subsection{Drug release mechanism}

In order to study the drug release mechanism from the fibers, various models were considered to fit the experimental data using Microsoft Excel software to execute and evaluate data modelling. The in vitro release data were evaluated to check the goodness of fit to Higuchi, Korsmeyer-Peppas, Linder-Lippold, Ritger-Peppas and Peppas-Sahlin models. All fitting models were performed by non-linear direct fitting and analyzed by the examination of the goodness of fit statistics such as the sum of squares due to error (SSE) and the adjusted Rsquare $\left(\mathrm{R}^{2}\right)$ by using a graph fitting software as an analysis tool.

\section{Results and discussion}

The main aim of this study was firstly to optimize procedure for electrospinning of beadfree alginate fibers loaded with ciprofloxacin hydrochloride and foremost formed with high content of sodium alginate. Secondly, to characterize them taking into account a drug release kinetics. To help readers follow the performed protocols we summarize all studied 
compositions of alginate fibers in Table 1, indicating figures in which the obtained fibers are presented. The bold letters indicates the images that present bead-free fibers.

\section{Table 1}

Summary of the composition of studied fibers (all set of experiments).

\begin{tabular}{|c|c|c|c|c|c|}
\hline \multirow{2}{*}{$\begin{array}{l}\text { AL } \\
\mathrm{wt} \%\end{array}$} & \multicolumn{2}{|c|}{ PEO } & \multirow[t]{2}{*}{ Surfactant } & \multirow{2}{*}{$\begin{array}{c}\text { CpHCl } \\
\text { wt\% }\end{array}$} & \multirow[t]{2}{*}{ Figure } \\
\hline & MW & $w t \%$ & & & \\
\hline \multirow[t]{7}{*}{2.0} & 100 & 2.0 & - & - & $1 \mathrm{~A}$ \\
\hline & 600 & 2.0 & - & - & $1 \mathrm{~B}$ \\
\hline & 1000 & 2.0 & - & - & $1 \mathrm{C}$ \\
\hline & & & 1.0 wt\% Pluronic & - & $4 \mathrm{~A}$ \\
\hline & 2000 & 2.0 & - & - & 1D, 2D \\
\hline & & & $0.1 \mathrm{wt} \%$ Triton & - & $2 \mathbf{E}$ \\
\hline & & & $0.1 \mathrm{wt} \%$ Pluronic & - & $2 F$ \\
\hline \multirow[t]{8}{*}{3.0} & 1000 & 1.5 & 1.0 wt\% Pluronic & - & $3 \mathrm{~A}$ \\
\hline & & 2 & - & - & $2 \mathrm{~A}$ \\
\hline & & & 0.5 wt\% Triton & - & 2B \\
\hline & & & 0.5 wt\% Pluronic & - & $2 \mathrm{C}, 3 \mathrm{~B}$ \\
\hline & & & 1.0 wt\% Pluronic & - & $3 C, 4 B$ \\
\hline & & & & 0.02 & 8A \\
\hline & & & & 0.08 & 8B \\
\hline & & & & 0.10 & $6 A, 8 C$ \\
\hline \multirow[t]{3}{*}{4.0} & 1000 & 1.5 & 1.0 wt\% Pluronic & - & $3 \mathrm{D}$ \\
\hline & & 2 & 0.5 wt\% Pluronic & - & $3 E$ \\
\hline & & & 1.0 wt\% Pluronic & - & $3 F, 4 C$ \\
\hline
\end{tabular}




\begin{tabular}{llllcc}
\hline & & $1.0 \mathrm{wt} \%$ Pluronic & 0.10 & $\mathbf{6 B}, 7 \mathrm{~A}, 7 \mathbf{7}$ \\
\hline 5.0 & 1000 & 2 & $1.0 \mathrm{wt} \%$ Pluronic & - & $4 \mathrm{D}$ \\
\hline
\end{tabular}

The presence of beads in the fibers is indicative of an unstable process [Siavash S. et al. Polymer Eng. Sci. 55 (2015) 2576-2582], as consequence the reproducibility of the obtained material is very low. Besides, only well-defined bead-free fibers can assure slow and sustained kinetics of a drug release, so important parameter in case of treatment of the infected wounds. It is well-documented that a drug release kinetics for polymeric capsules and fibers is totally altered. First of all, morphology of these formulations offers different surface area to volume ratios - the higher value yielding the higher rate of drug release in the case of fibers (for fixed drug loading). However, both theoretical predictions and the experimental data are in excellent agreement that polymeric fibers (PLGA) exhibit slower and less efficient releasing of a drug in comparison with capsules [2]. Thus, one would expect that in the case of beaded fibers the profiles of drug release could be complicated and unpredictable.

\subsection{Effect of additional polymer on formation of electrospun alginate fibers}

Due to the high viscosity and high electrical conductivity of alginate solutions, formation of fibrous structures by electrospinning of pure alginate is really difficult and leads to generation of sprayed droplets or short fibers embedded with beads. Another cause that limited the electrospinnability of aqueous sodium alginate solutions is the high surface tension. To solve these problems different approaches were used (i) incorporation of an additional well-electrospinable polymer, (ii) application of surfactants or/and (iii) addition of co-solvent to alginate solution [23]. The first solution implies the use of flexible and uncharged synthetic polymers (e.g. PEO and PVA) that via the hydrogen bonds formed between alginate and these polymers, decrease the repulsive force among polyanionic molecules and facilitate the chain entanglement [12]. Beside, Saquing et al. found that PEO favorably reduces surface tension, which facilitates electrospinning [20]. PEO is a unique class of non-ionic water-soluble biodegradable biopolymer, that due to its excellent biocompatibility, biodegradability and very low toxicity is proposed for the use in many biomedical applications [7, 22]. What is also noteworthy, PEO is approved by Food and Drug Admininistration. 
Since it is known that the polymer molecular weight modify the solutions rheological properties and as consequence has influence in the fiber size and morphology [24] PEO with four different average molecular weights as a carrier polymer was tested. The initial attempt at electrospinning of alginate-PEO fibers resulted in droplets or beaded fibers but no uniform nanofibers (Fig. 1).

A

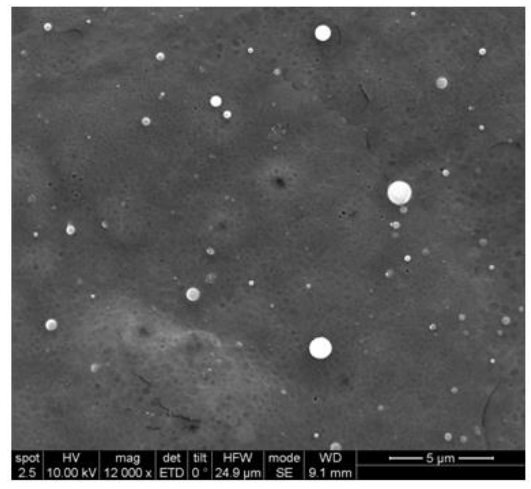

C

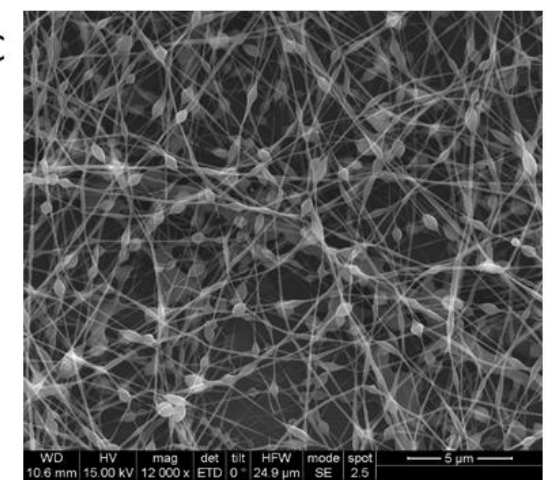

B

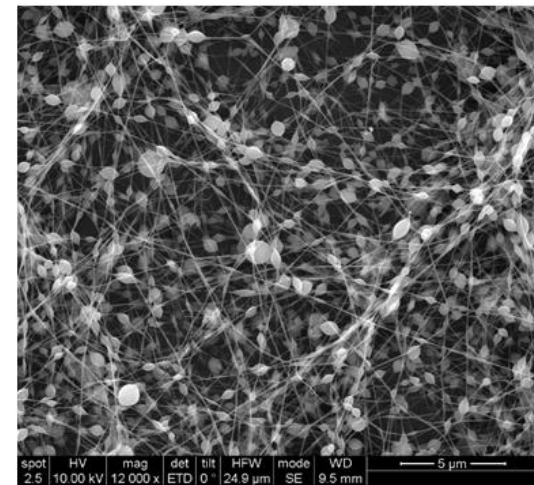

D

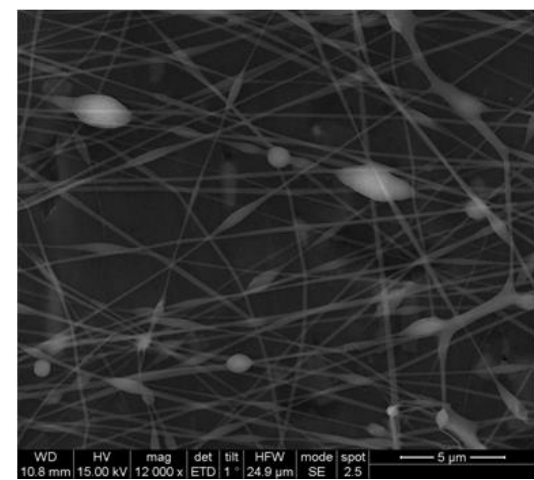

Fig. 1. SEM images of electrospun alginate fibers: a) 2.0 wt.\% AL, 2.0 wt.\% PEO $100 \mathrm{kDa}$, b) 2.0 wt.\% AL, 2.0 wt.\% PEO $600 \mathrm{kDa}$, c) 2.0 wt.\% AL, $2.0 \mathrm{wt} . \%$ PEO $1000 \mathrm{kDa}$ and d) 2.0 wt.\% AL, 2.0 wt.\% PEO 2000 kDa.

It was concluded that the higher molecular weight of the PEO better fibers with fewer beads are obtained. This is in agreement with work on the role of PEO as the "carrier polymer" in alginate-based nanofibers extensively studied by Saquing et al.. It was demonstrated that electrospining of alginate is only possible by blending with an appropriate polymer with a high molecular weight. It was speculated that PEO-PEO interactions of the high molecular weight producing sufficient chain entanglements in the resulting polymer blend solution play a key role in "carrying” the alginate from solution during electrospining [20]. 


\subsection{Effect of surfactant on formation of electrospun alginate fibers}

The second proposed solution of improvement of electrospinning from alginate solutions concerns addition of surfactant. Besides, the amount of alginate in the blend can be increased with the addition of small amounts of surfactant. A nonionic surfactant plays a important role to the mat formation during electrospinning process, especially influences on morphology of fibers. Reducing the surface tension and the suppression of bead's defects are two of the most important tasks that surfactants meet [22]. Triton X-100 and Pluronic F-127 are the most commonly used surfactants in electrospinning of alginate-based nanofibers. Both have hydrophilic PEO blocks, however the hydrophobic polypropylene oxide (PPO) block from Pluronic F-127 is less toxic than the alkyl benzene block in Triton X-100. For this reason Pluronic F-127 is a more viable material for biomedical applications and it is a FDAapproved surfactant [21, 22].

The addition of small amounts of Triton X-100 or Pluronic F-127 to the sample generates bead-free fibers when using PEO $1000 \mathrm{kDa}$, as shown in Fig. 2 (A-C). In cases of PEO with $\mathrm{M}_{\mathrm{W}} 2000 \mathrm{kDa}$ the addition of Triton X-100 produce fibers without beads but with very heterogeneous diameter. With the addition of Pluronic F-127 even more heterogeneous fibers were produced (Fig. 2 F). This could be related to the PEO surfactant interaction that modifies the surface tension of solutions leading to unfavorable electrospinning conditions. The surface tension was one of the most important parameters during the electrospinning process and it was previously reported that the polymer-surfactant interaction decrease the surface tension of solution [25]. Thus, in further study we decided to use PEO $1000 \mathrm{kDa}$. 
A

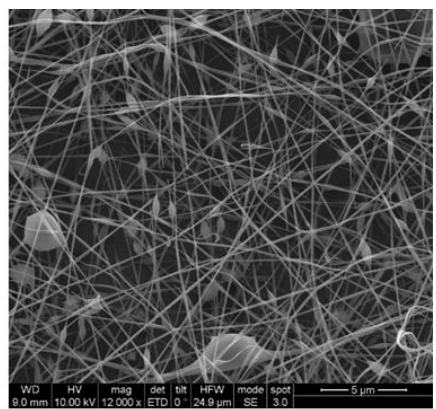

D

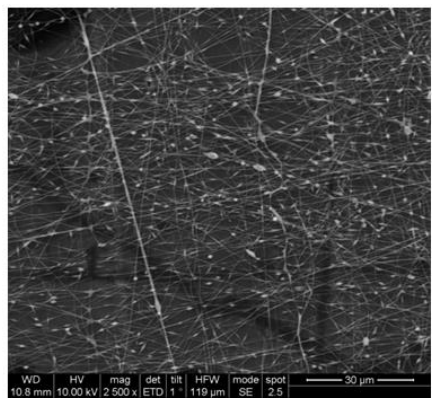

B

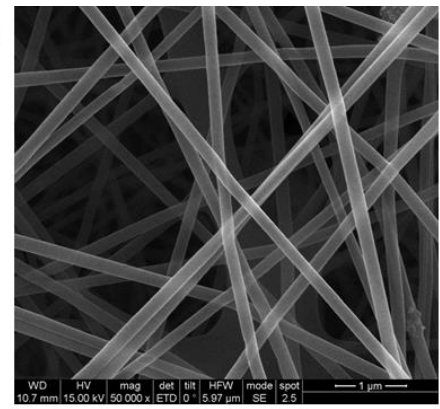

E

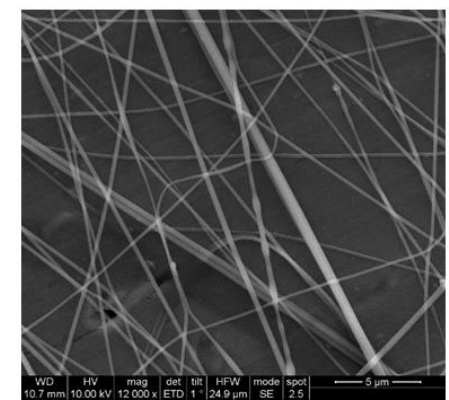

C

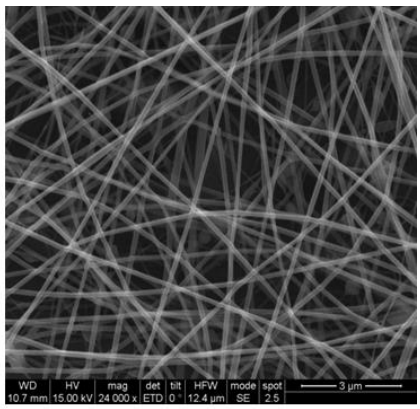

$\mathrm{F}$

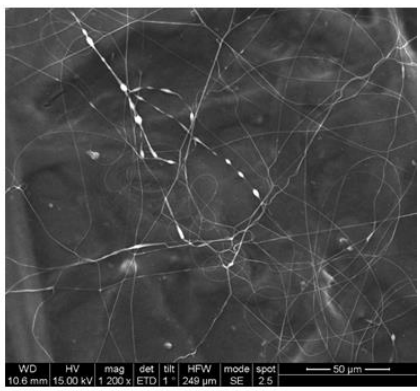

Fig. 2. SEM images of electrospun a) alginate fibers without surfactant (3.0 wt.\% AL, 2.0 wt.\% PEO $1000 \mathrm{kDa}$ ) and with addition of surfactant b) $0.5 \mathrm{wt} \%$ Triton X-100, c) 0.5 wt.\% Pluronic F-127 as well as d) alginate fibers without surfactant (2.0 wt.\% AL, 2.0 wt.\% PEO $2000 \mathrm{kDa}$ ) and with addition of surfactant e) $0.1 \mathrm{wt} \%$ Triton X-100, f) 0.1 wt.\% Pluronic F-127.

Since the potential use of alginate-based nanofibers is biomedical applications, there is a strong need to use only non-toxic reagents. Therefore in further study, we focused on application of Pluronic F-127 as a main surfactant that effectively suppresses formation of bead defects in the investigated systems (Fig. 3). 


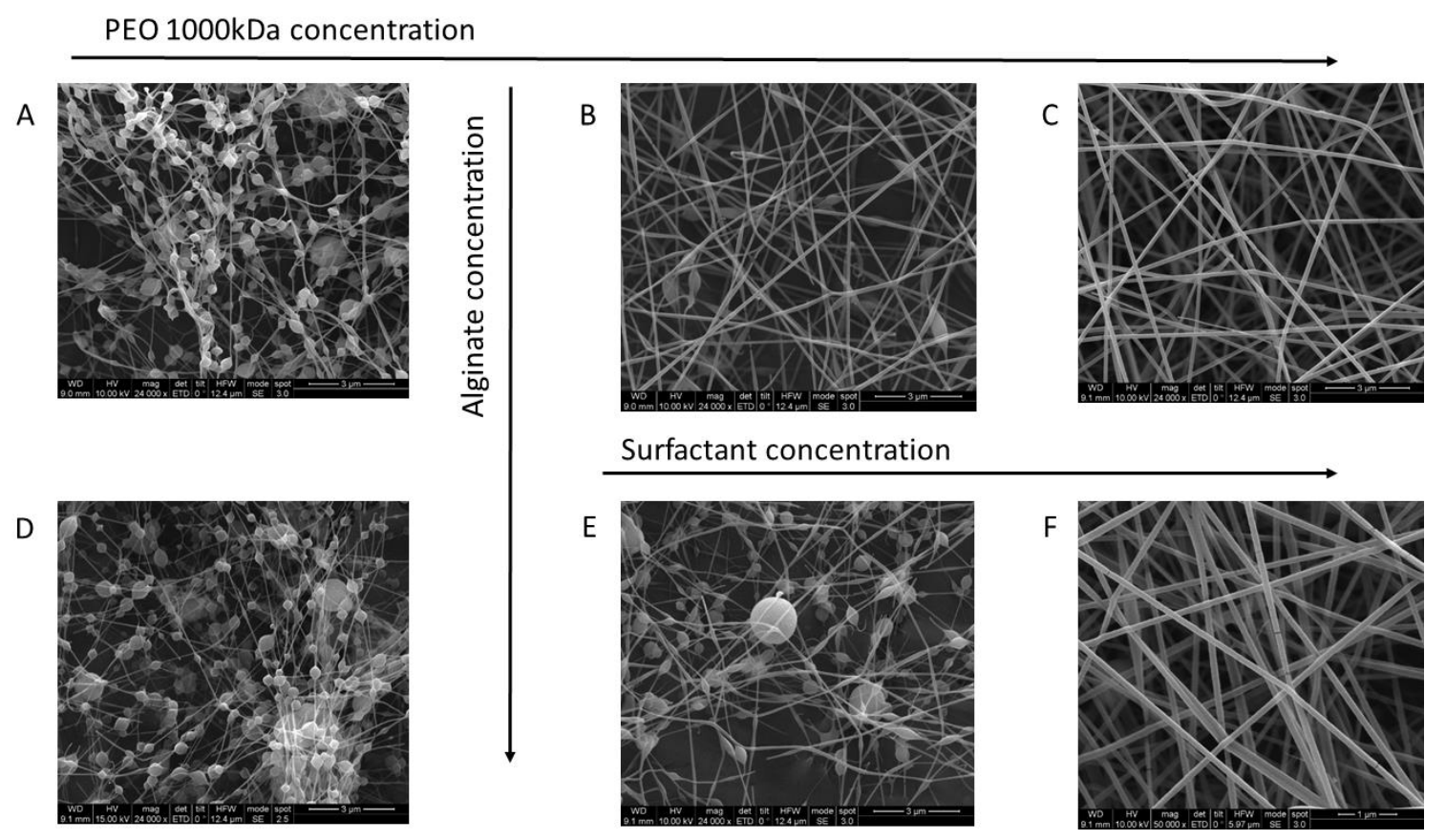

Fig. 3. SEM images of electrospun alginate fibers: a) 3.0 wt.\% AL, $1.5 \mathrm{wt} \%$ PEO $1000 \mathrm{kDa}$, 1.0 wt.\% Pluronic F-127, b) 3.0 wt.\% AL, 2.0 wt.\% PEO 1000 kDa, 0.5 wt.\% Pluronic F-127, c) 3.0 wt.\% AL, 2.0 wt.\% PEO 1000 kDa, 1.0 wt.\% Pluronic F-127, d) 4.0 wt.\% AL, 1.5 wt.\% PEO 1000 kDa, 1.0 wt.\% Pluronic F-127, e) 4.0 wt.\% AL, 2.0 wt.\% PEO 1000 kDa, 0.5 wt.\% Pluronic F-127, f) 4.0 wt.\% AL, 2.0 wt.\% PEO 1000 kDa, 1.0 wt.\% Pluronic F-127.

Free from beads and defects nanofibers with higher content of sodium alginate than additional polymer and surfactant have been successfully obtained only in case of $3.0 \mathrm{wt} . \%$ AL, 2.0 wt.\% PEO 1000 kDa, 1.0 wt.\% Pluronic F-127 (Fig. 3C) and 4.0 wt.\% AL, 2.0 wt.\% PEO 1000 kDa, 1.0 wt.\% Pluronic F-127 (Fig. 3F). They exhibited cylindrical shape and regular morphology with average diameter of $136 \pm 33 \mathrm{~nm}$ and $148 \pm 30 \mathrm{~nm}$ in case of lower and higher content of AL, respectively. Only simultaneous incorporation of the additional polymer and the surfactant resulted in nanofibers with perfect uniformity and structural integrity. Increase of only one of these components in blend alginate solution did not assure formation of bead-free fibers (Fig. 3). Thus, both well-electrospinable polymer (PEO) and a surfactant (in general, Triton X-100 or Pluronic F-127) have a substantial effect on electrospinning processability of alginate nanofibers.

Our findings concerning the important role of surfactant in formation of uniform nanofibers with high content of alginate are in agreement with other authors [20-22]. For instance, Bonino et al. as well concluded that the addition of small amount of a surfactant 
(1.0 wt.\%) effectively lowers the surface tension of polymer solution, supports formation of uniform nanofibers and enhances concentration of alginate and morphology of electrospun fibers from alginate-PEO blend solutions [21].

\subsection{Influence of alginate concentration on alginate electrospun fibers formation}

Furthermore, influence of increasing alginate concentration and constant concentration of Pluronic F-127 (1.0 wt.\%) on formation of nanofibers with respectable uniformity and structural integrity has been investigated. SEM images of the electrospun fibers with increasing content of alginate are shown in Fig. 4.

A

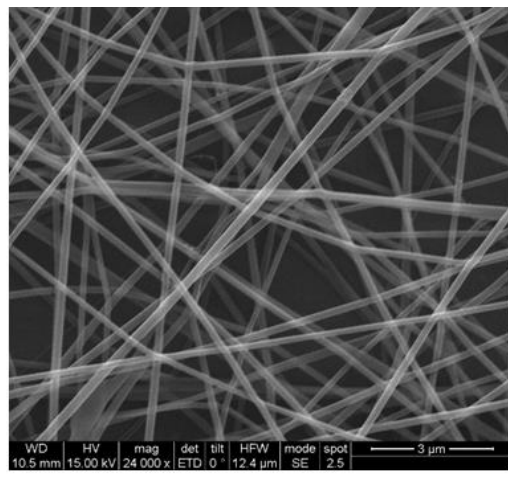

C

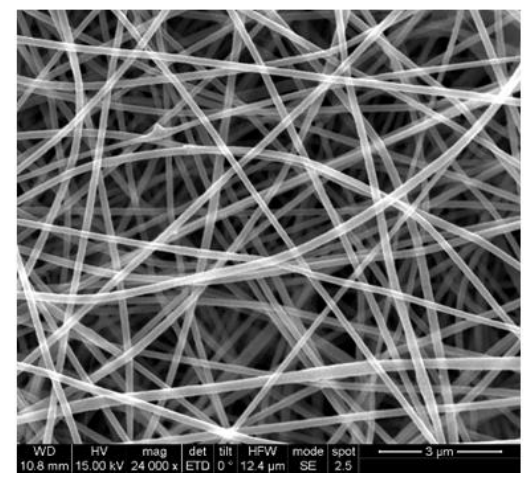

B

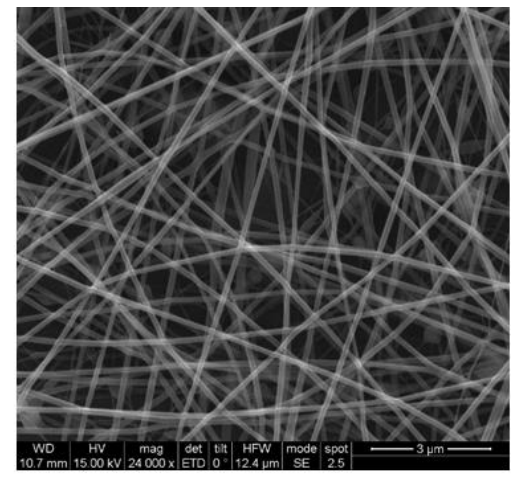

D

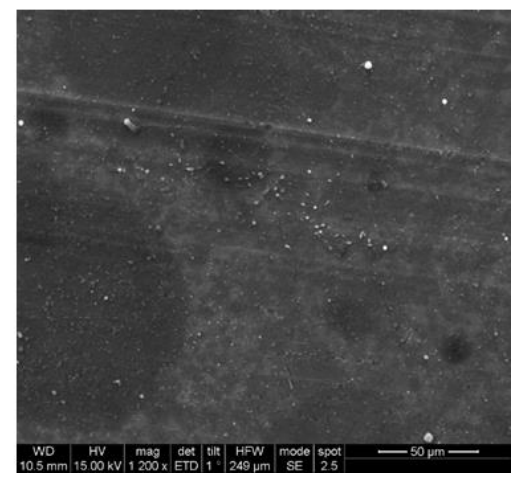

Fig. 4. SEM images of different electrospun alginate fibers: a) $2.0 \mathrm{wt} \% \mathrm{AL}, 2.0 \mathrm{wt} . \% \mathrm{PEO}$ 1000 kDa, 1.0 wt.\% Pluronic F-127, b) 3.0 wt.\% AL, 2.0 wt.\% PEO 1000 kDa, 1.0 wt.\% Pluronic F-127, c) 4.0 wt.\% AL, 2.0 wt.\% PEO 1000 kDa, 1.0 wt.\% Pluronic F-127, d) 5.0 wt.\% AL, 2.0 wt.\% PEO 1000 kDa, 1.0 wt.\% Pluronic F-127.

Smooth and uniform nanofibers were obtained in range of 2-4 wt.\% of sodium alginate with constant concentration of PEO $1000 \mathrm{kDa}$ (2.0 wt.\%) and Pluronic F-127 (1.0 wt.\%) 
(Fig. 4A-C). Increased alginate concentration resulted in no significant trend in nanofiber sizes changes (taking into account the determined standard deviation error). Average size calculated for approximately 200 fibers was estimated to be $125 \pm 29 \mathrm{~nm}, 109 \pm 24$ and $177 \pm 50$ $\mathrm{nm}$ for 2.0, 3.0 and 4.0 wt.\% AL, 2.0 wt.\% PEO $1000 \mathrm{kDa}, 1.0 \mathrm{wt}$ \% Pluronic F-127, respectively. Increase of alginate concentration to $5.0 \mathrm{wt}$ \% without changing of PEO $1000 \mathrm{kDa}$ and surfactant content resulted in droplets formation (Fig. 4D). It can be supposed that electrospinning was impossible because of high conductivity of alginate. This also confirms that addition of both PEO and surfactant is necessary to generate bead-free fibers.

\subsection{Crosslinking and stabilization process of unloaded fibers}

PEO, necessary to improve the electrospinnability of the alginate, is a non-toxic, biologically inert and water soluble polymer. However, according to the literature [26] it can be remove from the fiber during the crosslinking process. In order to confirm the removal of the PEO, ATR-FTIR was used to examine the ciprofloxacin unloaded fibers before and after the treatment. Pure PEO, Pluronic F-127 and alginate spectra were included as references (Fig. 5).

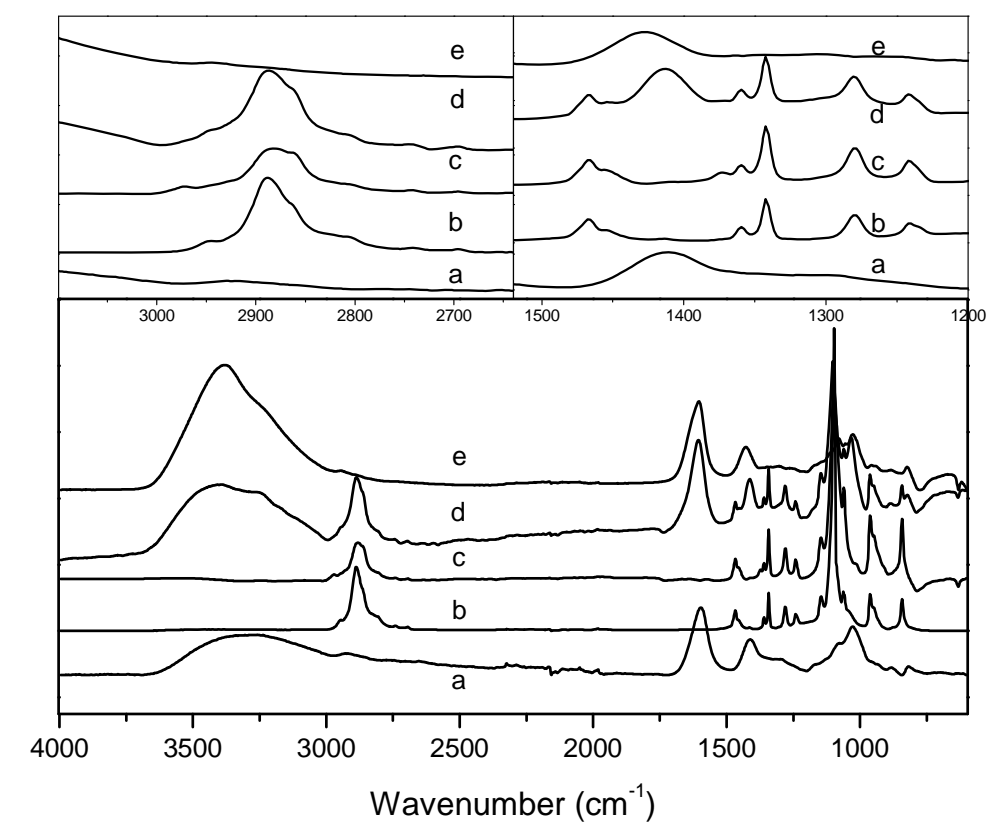


Fig. 5. FTIR spectra of a) AL, b) PEO $1000 \mathrm{kDa}$, c) Pluronic P-127, d) untreated and e) treated 4.0 wt.\% AL, 2.0 wt.\% PEO 1000 kDa, 1.0 wt.\% Pluronic F-127 fibers.

AL spectrum shows a characteristic peaks at 1595 and $1407 \mathrm{~cm}^{-1}$ related to the asymmetric and symmetric stretching of $-\mathrm{COO}^{-}$groups, respectively, and the asymmetrical $\mathrm{C}-\mathrm{O}-\mathrm{C}$ stretching modes at $1029 \mathrm{~cm}^{-1}[4,27]$. While, PEO spectrum presents characteristic peaks at 2886, 1100 and $962 \mathrm{~cm}^{-1}$ related to the stretching, rocking and twisting of the methylene group. The band at $842 \mathrm{~cm}^{-1}$ can be assigned to the stretching vibration of $\mathrm{C}-\mathrm{O}-\mathrm{C}$ and $\mathrm{C}-\mathrm{C}$ molecular bonds [28]. Similar spectrum was obtained for Pluronic F-127 since it is a triblock copolymer of PEO and polyproylene oxide [29]. As expected the spectrum of electrospun fibers before treatment exhibited all these peaks confirming the presence of PEO in the fibers. However, after treatment these peaks were no longer present in the fiber spectrum, in agreement with the complete removal of PEO and Pluronic F-127 from the fibers. Besides, there is a shift of the peak related to symmetric stretching of $-\mathrm{COO}^{-}$groups to higher frequency $1604 \mathrm{~cm}^{-1}$ that could be attributed to an increase of the interaction between these groups of alginate and $\mathrm{Ca}^{2+}$ ions [11].

\subsection{Formation of alginate electrospun fibers loaded with ciprofloxacin hydrochloride}

Mixing antibiotics in the polymer solution prior to electrospinning is an easy method to load large quantities of a drug into practically any polymeric nanofibers. However, there are disadvantages of the resulting systems, mainly the loaded drug tends to leach out rapidly from the fibrous mats in an aqueous solution. This effect is termed "burst release" and is widely described in scientific literature [2, 30-32].

Using the same procedure as described in the previous sections, alginate-PEO blended solutions with the addition of ciprofloxacin hydrochloride were electrospun (Fig. 6). 

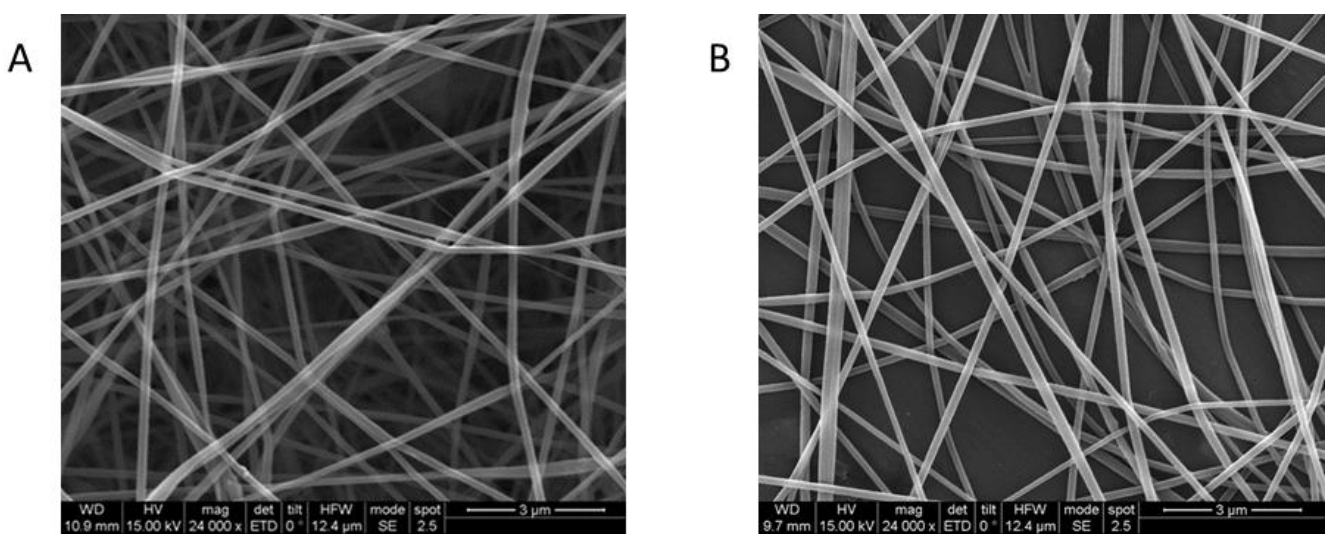

Fig. 6. SEM images of different electrospun alginate fibers loaded with ciprofloxacin: a) 3.0 wt.\% AL, 2.0 wt.\% PEO 1000 kDa, 1.0 wt.\% Pluronic F-127, 0.1 wt.\% CpHCl (1.0 mg/ml) and b) $4.0 \mathrm{wt} \%$ AL, $2.0 \mathrm{wt} \%$ PEO $1000 \mathrm{kDa}, 1.0 \mathrm{wt} . \%$ Pluronic F-127, 0.1 wt.\% CpHCl (1.0 mg/ml).

The resulting nanofibers after addition of $\mathrm{CpHCl}$ were smooth and without any beads. It was concluded that the addition of another component i.e. antibiotic to the electrospinning mixture did not changed significantly electrospinability of sodium alginate. Desirable smooth and flexible alginate fibers with addition of PEO $1000 \mathrm{kDa}$ (2.0 wt.\% PEO) and Pluronic F127 (1.0 wt.\%) were obtained with the average size of $119 \pm 36 \mathrm{~nm}$ and $161 \pm 32 \mathrm{~nm}$ for 3.0 and 4.0 wt.\% AL, respectively.

In general, the morphology of electrospun nanofibers depends on solution parameters such as conductivity, viscosity, and surface tension [6, 12, 22, 33]. When an aqueous drug solution is added into a polymer solution, the viscosity and surface tension of the mixed solution alter slightly, usually overall effect is negligible. Meanwhile, the conductivity of the solution can be increased by the presence of ionized drug molecules (although the exact value of increase in conductivity is not known), which increases the charge density of the jet resulting in the fibrous morphology [2, 34-36]. In our case the electrospinning process was possible and resulted in formation of regular fibers

\subsection{Post-treatment - stabilization and cross-linking of loaded fibers}

As expected, the as-prepared nanofibers were highly water-soluble and dissolved in water-based media. Therefore, to improve the stability of the electrospun mats they were 
immersed in ethanol for $1 \mathrm{~min}$ and then in ethanol solution of $\mathrm{CaCl}_{2}$ for $10 \mathrm{~min}$. Consequently, stabilization and cross-linking processes occurred in these conditions. The electrospun alginate fibers before and after post-treatment are presented in Fig. 7.

A

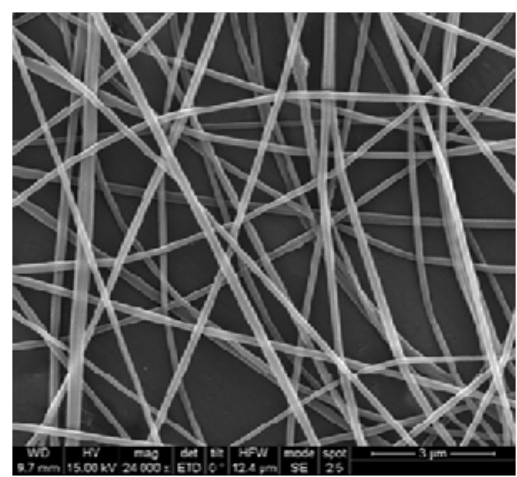

B

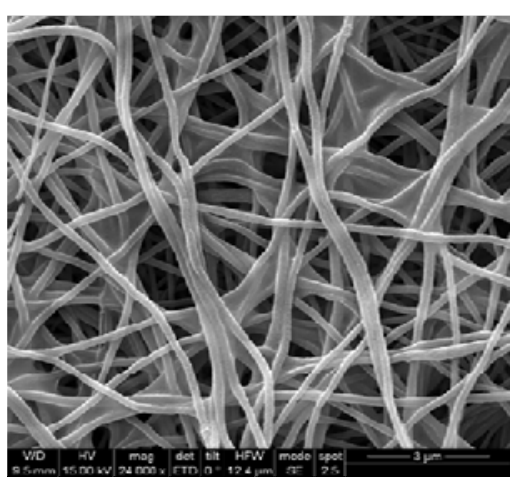

Fig. 7. SEM images of electrospun alginate fibers loaded with ciprofloxacin $4.0 \mathrm{wt} \% \mathrm{AL}$, 2.0 wt.\% PEO 1000 kDa, 1.0 wt.\% Pluronic F-127, 0.1 wt.\% CpHCl (1mg/ml) a) before and b) after stabilization and cross-linking processes.

The morphology of the stabilized and cross-linked alginate nanofibers changed slightly after applied post-treatment. They puffed up and interfused inconsiderably, however, what is noteworthy, the nanofibrous structure was maintained. The increase in the fibers' diameter from $161 \pm 32 \mathrm{~nm}$ to $181 \pm 45 \mathrm{~nm}$ was the only noticeable effect that was observed after treatment procedure. The increase in diameter has been also observed by other authors and was attributed to the swelling during PEO dissolution [37]. It was concluded that the alginate-based nanofibers were successfully ionically cross-linked in a calcium solution without the need of the cytotoxic chemical cross-linkers application (e.g., glutaraldehyde). Determined diameter of obtained alginate fibers is in agreement with that published by other authors. For example, Bonino et al. obtained uniform nanofibers containing alginate, PEO (600 kDa) and Pluronic F-127 with the average fiber diameters $150 \mathrm{~nm}$, independently on the molecular weight of used alginate (37 and $196 \mathrm{kDa}$ ). Moreover, the authors speculate that the final fiber diameter is not dependent on solution composition but rather on the similarities in the solution storage and processing conditions such as viscosity, temperature, and humidity [21]. The above-mentioned speculations were confirmed by these authors, who showed the influence of relative humidity on diameter of electrospun fibers (blends of alginate (37 kDa), PEO (600 kDa), Pluronic F-127 as surfactant). It was concluded that the increased relative humidity results in decrease of fiber size [22]. 


\subsection{Loading efficiency}

For nanofibers with $3.0 \mathrm{wt} . \%$ of alginate mixed with $2.0 \mathrm{wt} . \%$ of PEO $1000 \mathrm{kDa}$ and 1 wt.\% of surfactant (Pluronic F-127) it was possible to load even $1.0 \mathrm{mg} / \mathrm{ml}$ of $\mathrm{CpHCl}$ in the precursor solution without any adverse effect on the structure, morphology and size of the resulting nanofibers (Fig. 8). According with these results and in order to have the maximum ciprofloxacin load in the fibers, the materials hereafter were prepared using $1.0 \mathrm{mg} / \mathrm{ml}$ of CpHCl.

A

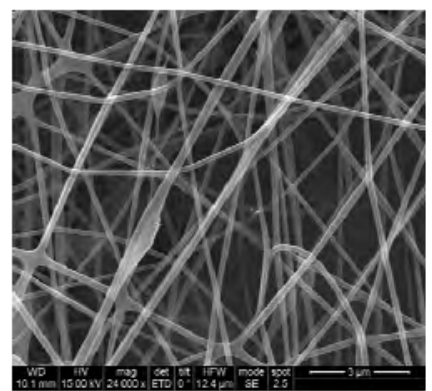

B

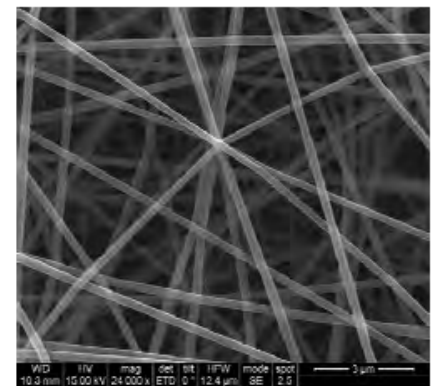

C

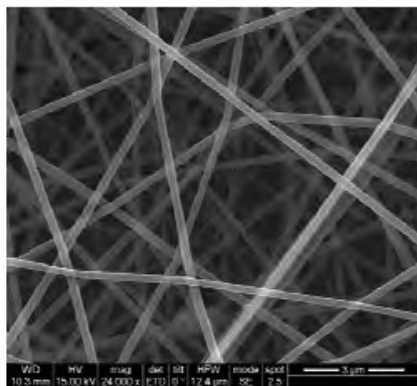

Fig. 8. SEM images of as-electrospun alginate fibers loaded with different concentration of ciprofloxacin hydrochloride: a) 3.0 wt.\% AL, 2.0 wt.\% PEO 1000 kDa, 1.0 wt.\% Pluronic F127, 0.02 wt.\% CpHCl (0.2 mg/ml), b) 3.0 wt.\% AL, 2.0 wt.\% PEO 1000 kDa, 1.0 wt.\% Pluronic F-127, 0.08 wt.\% CpHCl (0.8 mg/ml) and c) 3.0 wt.\% AL, 2.0 wt.\% PEO 1000 kDa, 1.0 wt.\% Pluronic F-127, 0.1 wt.\% CpHCl (1.0 mg/ml).

The amount of ciprofloxacin measured in the as-spun fibers (3.0 wt.\% AL, 2.0 wt.\% PEO $1000 \mathrm{kDa}, \quad 1.0 \mathrm{wt} \% \quad$ Pluronic F-127, $\quad 0.1 \mathrm{wt} \% \quad \mathrm{CpHCl} \quad(1.0 \mathrm{mg} / \mathrm{ml})) \quad$ was $1.13 \pm 0.02$ wt. $\%$ implying a LE value of $82.7 \pm 1.5 \%$. In order to known the drug loading after stabilization and crosslinking processes, the amount of $\mathrm{CpHCl}$ in crosslinking and stabilization solutions was quantify. It was calculated that $61.6 \pm 6.9 \%$ of the drug present in the as-spun fibers was lost during the post-treatment. As consequence the final fibers contain $1.21 \pm 0.16$ wt.\% ciprofloxacin (assuming that all PEO and Pluronic F-127 were eliminated during the crosslinking process), what gives a LE for the complete process of $51.0 \pm 6.7 \%$.

\subsection{Release of ciprofloxacin hydrochloride}


In general, in case of nanofibers electrospun in such a way that the drug is mixed with polymer prior to manufacturing, the release profile in an aqueous environment is found to have a biphasic kinetics. An initial burst release is usually followed by a much slower second process. The high burst release can be ascribed mainly to the fact that the small diameter and the high surface area in the nanofibers guarantee short diffusion pathway and are favorable to mass transfer of the drug [2, 35, 36].

Fig. 9 shows the profile of $\mathrm{CpHCl}$ release from fibers, expressed as cumulative percentage of drug release versus time.

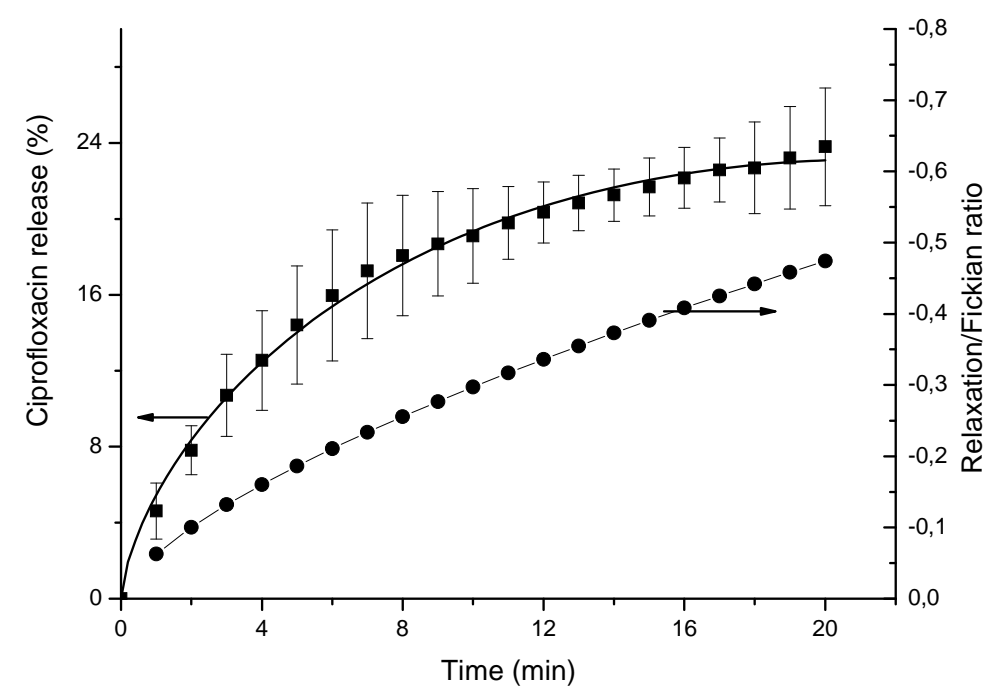

Fig. 9. Ciprofloxacin hydrochloride cumulative percent release profile and Peppas-Sahlin model fitting and $\mathrm{R} / \mathrm{F}$ ratio profile. In the case of drug release profile a standard deviation for each time point is given.

After 20 h only around $24 \%$ of the total loaded drug was released suggesting that the rest of $\mathrm{CpHCl}$ is still inside the fibers. The slightly increasing slope of the curve suggests that the releasing would continue after that time, but the amount of drug in the media was undetectable by our UV-Vis equipment. This behavior is different from the reported by Sibaja et al. [38] for alginate-chitosan fibers that release about $40-50 \%$ of the drug in the first hour. It would indicate that the crosslinking and stabilization processes avoid the fast drug release in the prepared AL fibers. As well, Hajiali et al. [4] demonstrated that with increasing the duration time of the post-treatment with trifluoroacetic acid, degradation rate of alginate nanofibers is prolonged. Thus, any strategy to enhance the stability and to control 
degradability of the broad utilized electrospun sodium alginate fibers is highly required, since application of these polymeric scaffolds, in for instance tissue engineering, is now strongly limited by their high solubility and the difficulty in adjustment of degradation dynamic and a drug release kinetics. What is noteworthy, the obtained by us release profile is similar to the one obtained for crosslinked sodium alginate/PEO hydrogel membranes by Mallikarjuna et al. [27]. However, in the case of our fibers after 20 hours only about $24 \%$ was released, while for hydrogels less than 14 hours were necessary to achieve $60 \%$ drug release in the best case.

Several models were used to evaluate the release kinetics (results not shown) but Peppas-Sahlin model was the only one that fits for the $\mathrm{CpHCl}$ release data giving good $\mathrm{R}^{2}$ values:

$\frac{M_{t}}{M_{\infty}}=k_{1} t^{n}+k_{2} t^{2 n}$

where $k_{1}, k_{2}$, and $n$ are constants, $M_{t} / M_{\infty}$ is the drug release fraction at time t. The first term on the right-hand side represents the Fickian diffusional contribution $(F)$ and the second one is the case-II relaxational contribution (R) [39]. The obtained parameters are shown in Table 2; the $\mathrm{R}^{2}$ values for the rest of models were lower than 0.985 .

\section{Table 2}

Peppas-Sahlin parameters.

\begin{tabular}{|c||c||}
\hline \hline \multicolumn{2}{|c|}{ Peppas-Sahlin parameters } \\
\hline \hline $\mathrm{k}_{1}$ & $5.82 \pm 0.25$ \\
\hline \hline $\mathrm{k}_{2}$ & $-0.36 \pm 0.03$ \\
\hline \hline $\mathrm{n}$ & $0.67 \pm 0.02$ \\
\hline \hline $\mathrm{R}^{2}$ & 0.995 \\
\hline
\end{tabular}

The obtained $n$ value is in the range reported for cylinders $(0.45<\mathrm{n}<0.89)$ with combined transport mechanism [40]. The fact that $\mathrm{k}_{1}$ is higher than $\mathrm{k}_{2}$ indicates that Fickian diffusion makes the predominant contribution to ciprofloxacin hydrochloride from alginate fibers release [41, 42]. The negative value obtained for $k_{2}$ should be interpreted in terms of a relaxation/dissolution mechanism insignificant compared to the diffusion process. Alginate is a highly soluble compound, but after the cross-linking process the dissolution would be avoided and the fibers entanglement would hinder the relaxation. The R/F profile provides information about the ratio of magnitude of diffusion due to polymer relaxation and Fickian 
diffusion. In other words, R/F profiles having values $>1$ suggest polymer relaxation-mediated diffusion is predominant as compared to the Fickian diffusion. In our case the R/F ratio profile (Fig. 9), calculated using the equation $R / F=\left(k_{2} / k_{1}\right) t^{n}$, is lower than 1 in all the studied range $[42,43]$ indicating also the predominance of the Fickian diffusion. It is interesting to see that with the increase in the experimental time there was an increase in the polymer relaxation/diffusion ratio. It was expected that after several minutes of immersion in PBS some relaxation processes start even in the crosslinked fibers. The obtained Peppas-Sahlin parameters confirm that the $\mathrm{CpHCl}$ release follows a diffusion-controlled mechanism.

\section{Conclusions}

The resulting nanofibers were formed with high content of sodium alginate, a natural highly biocompatible polysaccharide, as well as an addition of PEO and Pluronic F-127 (an ethylene oxide(EO)/propylene oxide (PO) block copolymer), both Food and Drug Administration approved polymers. What is noteworthy, a carrier polymer (PEO) was completely removed from alginate fibers during their crosslinking with calcium ions and stabilization process in ethanol. Stable alginate nanofibers loaded with ciprofloxacin hydrochloride revealed the average diameter of $109 \mathrm{~nm}$ and $161 \mathrm{~nm}$ for unloaded and loaded fibers, respectively. Loading efficiency was determined to be $51 \%$. While, the study on the release kinetics proved the combined transport mechanism with the predominant contribution of Fickian diffusion. The use of biopolymers as well as non-toxic solvents and crosslinkers makes the resultant scaffolds promising for various biomedical applications such as wound healing, regenerative medicine and drug delivery systems. It can be also supposed that since the produced alginate scaffolds are free from cytotoxic chemicals and possess appropriate structural properties they can promote the attachment and proliferation of cells.

\section{Acknowledgements}

This work was supported by Foundation for Polish Science within POMOST project “Alginate/chitosan core-shell beads with bioactive functionalities” (POMOST/2013-7/7).

\section{Abbreviations}


$\mathrm{AL}$ - sodium alginate

$\mathrm{CpHCl}$ - ciprofloxacin hydrochloride

FDA - Food and Drug Administration

PCL - polycaprolactone,

PLA - polylactic acid,

PGLA - poly(lactic-co-glycolic acid),

PEO - polyethylene glycol, poly(ethylene oxide),

PVA - poly(vinyl alcohol),

PVP - poli(vinylpyrrolidone),

ECMs - extracellular matrix,

\section{References}

1. Stachewicz U, Qiao T, Rawlinson FCS, Veiga Almeida F, Li WQ, Cattell M, and Barber AH. Acta Biomaterialia 2015;27:88-100.

2. Fattahi P, Borhan A, and Abidian MR. Advanced Materials 2013;25(33):4555-4560.

3. Qi H, Hu P, Xu J, and Wang A. Biomacromolecules 2006;7:2327-2330.

4. Hajiali H, Heredia-Guerrero JA, Liakos I, Athanassiou A, and Mele E. Biomacromolecules 2015;16:936-943.

5. Ma G, Fang D, Liu Y, Zhu X, and Nie J. Carbohydrate Polymers 2012;87:737- 743.

6. Lu JL, Zhu YL, Guo ZX, Hu P, and Yu J. Polymer 2006;47:8026-8031.

7. Bhattarai N, Li Z, Edmondson D, and Zhang M. Adv. Mater. 2006;18:1463-1467.

8. Lee KY, Jeong L, Kang YO, Lee SJ, and Park WH. Advanced Drug Delivery Reviews 2009 61:1020-1032.

9. Thet NT, Alves DR, Bean JE, Booth S, Nzakizwanayo J, Young AER, Jones BV, and Jenkins ATA. ACS Appl. Mater. Interfaces 2016;8:14909-14919.

10. Santoro M, Shah SR, Walker JL, and Mikos AG. Advanced Drug Delivery Reviews 2016;107:206-212.

11. Guiping M, Dawei F, Yang L, Zhu X, and Nie J. Carbohydrate Polymers 2012;87:737-743.

12. Hu X, Liu S, Zhou G, Huang Y, Xie Z, and Jing X. Journal of Controlled Release 2014;185:12-21.

13. Kataria K, Gupta A, Rath G, Mathur RB, and Dhakate SR. International Journal of Pharmaceutics 2014;469:102-110.

14. Unnithan AR, Barakat NAM, Pichiah PBT, Gnanasekaran G, Nirmala R, Cha YS, Jung CH, ElNewehy M, and Kim HY. Carbohydrate Polymers 2012;90:1786- 1793.

15. Watthanaphanit A, Supaphol P, Furuike T, Tokura S, Tamura H, and Rujiravanit R. Biomacromolecules 2009;10:320-327.

16. Paneva D, Ignatova M, Manolova N, and Rashkov I. Novel chitosan-containing micro- and nanofibrous maerials by electrospinning: preparation and biomedical application: Nova Science Publishers, Inc., 2009.

17. Zamani M, Prabhakaran MP, and Ramakrishna S. International Journal of Nanomedicine 2013;3(8):2997-3017.

18. Lee KY and Mooney D, J. Progress in Polymer Science 2012;37:106- 126. 
19. Sun J and Tan H. Materials 2013;6:1285-1309.

20. Saquing CD, Tang C, Monian B, Bonino CA, Manasco JL, Alsberg E, and Khan SA. Ind. Eng. Chem. Res. 2013;52:8692-8704.

21. Bonino CA, Krebs MD, Saquing CD, Jeong SI, Shearer KL, Alsberg E, and Khan SA. Carbohydrate Polymers 2011;85:111-119.

22. Bonino CA, Efimenko K, Jeong SI, Krebs MD, Alsberg E, and Khan SA. Small 2012:1-9.

23. Gonen SO, Taygun ME, and Kucukbayrak S. Materials Science and Engineering: $C$ 2016;58:709-723.

24. Rieger KA, Birch NP, and Schiffman JD. Carbohydrate Polymers 2016;139:131-138.

25. Zhao J, Sun Z, Shao Z, and Xu L. Fibers and Polymers 2016;17(6):896-901.

26. Jeong SI, Krebs MD, Bonino CA, Khan SA, and Alsberg E. Macromolecular Bioscience 2010;8(10):934-943.

27. Mallikarjuna B, Rao KM, Siraj S, Babu AC, Rao CK, and Subha MCS. Designed Monomers and Polymers 2013;16:151-159.

28. Colin-Orozco J, Zapata-Torres M, Rodriguez-Gattorno G, and Pedroza-Islas R. Food Biophysics 2015;10:134-144.

29. Cabana A, Ait-Kadi A, and Juhasz J. Journal of Colloid and Interface Science 1997;190:307312.

30. Sultanova Z, Kaleli G, Kabay G, and Mutlu M. International Journal of Pharmaceutics 2016;505:133-138.

31. Sperling LE, Reis KP, Pranke P, and Wendorff JH. Drug Discovery Today 2016;21(8):12431256.

32. Son YJ, Kim WJ, and Yoo HS. Arch. Pharm. Res. 2014;37:69-78.

33. Gupta NR, Prasad BLV, Gopinath CS, and Badiger MV. Rsc Advances 2014;4(20):10261-10268.

34. Pillay V, Dott C, Choonara YE, Tyagi C, Tomar L, Kumar P, du Toit LC, and Ndesendo VMK. Journal of Nanomaterials 2013:1-22.

35. Gao Y, Truong YB, Zhu Y, and Kyratzis IL. J. Appl. Polym. Sci. 2014:40797-40810.

36. Khalf $A$ and Madihally SV. European Journal of Pharmaceutics and Biopharmaceutics 2017;112:1-17.

37. Mokhena TC and Luy AS. Journal of Cleaner Production 2017;156:470-479.

38. Sibaja B, Culbertson E, Marshall P, Boy R, Broughton RM, Solano AA, Esquivel M, Parker J, De La Fuente L, and Auad ML. Carbohydrate Polymers 2015;134:598-608.

39. Siepmann J and Peppas NA. Advanced Drug Delivery Reviews 2012;64:163-174.

40. Peppas NA and Sahlin JJ. International Journal of Pharmaceutics 1989;57(2):169-172.

41. Lupo B, Maestro A, Gutiérrez JM, and González C. Food Hydrocolloids 2015;49:25-34.

42. Aragon J, Gonzalez R, Fuentes G, Palin L, Croce G, and Viterbo D. Science and Engineering of Composite Materials 2010;17(3):173-182.

43. Yadav I, Shaw GS, Nayak SK, Banerjee I, Shaikh H, AI-Zahrani SM, Anis A, and Pal K. Iranian Polymer Journal 2016;25(9):799-810. 